\title{
Continuous positive airway pressure (CPAP) vs noninvasive positive pressure ventilation (NIPPV) vs noninvasive high frequency oscillation ventilation (NHFOV) as post- extubation support in preterm neonates: protocol for an assessor-blinded, multicenter, randomized controlled trial
}

Yuan Shi ${ }^{1+}$, Daniele De Luca ${ }^{2,3,4^{*+}}$ (D) and for the NASal OscillatioN post-Extubation (NASONE) study group

\begin{abstract}
Background: Various noninvasive respiratory support modalities are available in neonatal critical care in order to minimize invasive ventilation. Continuous positive airway pressure (CPAP) is the more commonly used but noninvasive positive pressure ventilation (NIPPV) seems more efficacious in the early post-extubation phase, although it is not clear if NIPPV may influence longterm outcomes. A recently introduced alternative is noninvasive high frequency oscillatory ventilation (NHFOV) which might be especially useful in babies needing high constant distending pressure. Preterm neonates may receive these respiratory supports for several weeks. Nonetheless, no data are available for the longterm use of NIPPV and NHFOV; few data exist on NHFOV and clinical outcomes, although its safety and suitability are reported in a number of preliminary short-term studies.

Methods: We designed an assessor-blinded, multicenter, three-arms, parallel, pragmatic, randomized, controlled trial with a superiority design, investigating the use of CPAP vs NIPPV vs NHFOV during the whole stay in neonatal intensive care units in China. Since safety data will also be analyzed it may be considered a phase II/III trial. Moreover, subgroup analyses will be performed on patients according to prespecified criteria based on physiopathology traits: these subgroup analyses should be considered preliminary. At least 1440 neonates are supposed to be enrolled. The trial has been designed with the collaboration of international colleagues expert in NHFOV, who will also perform an interim analysis at the about $50 \%$ of the enrolment.

\footnotetext{
* Correspondence: dm.deluca@icloud.com

${ }^{\dagger}$ Yuan Shi and Daniele De Luca contributed equally to this work.

2Division of Pediatrics and Neonatal Critical Care, "A.Béclère" Medical Center,

South Paris University Hospitals, AP-HP, Paris, France

${ }^{3}$ Physiopathology and Therapeutic Innovation Unit-INSERM U999, South

Paris-Saclay University, Paris, France

Full list of author information is available at the end of the article
}

(c) The Author(s). 2019 Open Access This article is distributed under the terms of the Creative Commons Attribution 4.0 International License (http://creativecommons.org/licenses/by/4.0/), which permits unrestricted use, distribution, and reproduction in any medium, provided you give appropriate credit to the original author(s) and the source, provide a link to the Creative Commons license, and indicate if changes were made. The Creative Commons Public Domain Dedication waiver (http://creativecommons.org/publicdomain/zero/1.0/) applies to the data made available in this article, unless otherwise stated. 
(Continued from previous page)

Discussion: The study is applying the best trial methodology to neonatal ventilation, a field where it is often difficult to do so for practical reasons. Nonetheless, ours is also a physiology-driven trial, since interventions are applied based on physiological perspective, in order to use ventilatory techniques at their best. The pragmatic design will increase generalizability of our results but subgroup analyses according to predefined physiopathological criteria are also previewed trying to have some advantages of an explanatory design. Since not all clinicians are well versed in all respiratory techniques, the training is pivotal. We intend to apply particular care to train the participating units: a specific 3-month period and several means have been dedicated to this end.

Trial registration: NCT03181958 (registered on June 9, 2017).

Keywords: Neonate, Noninvasive respiratory ventilation, Non invasive high frequency oscillatory ventilation

\section{Background}

\section{General background}

Respiratory distress syndrome (RDS) is the main cause of respiratory failure in preterm neonates, its incidence varying from $\approx 80 \%$ to $\approx 25 \%$ depending on gestational age [1]. When optimal prenatal care is provided, the best approach to treat RDS, according to several recent trials $[2,3]$, consists in providing continuous positive airway pressure (CPAP) from the first minutes of life using short binasal prongs or masks [4, 5], followed by early selective surfactant administration for babies with worsening oxygenation and/or increasing work of breathing. Any effort must be done to minimize the time under invasive mechanical ventilation (IMV) [6]. Nonetheless, clinical trials have shown that a relevant proportion of preterm neonates fails this approach and eventually need IMV during their hospitalization [7-9]. The duration of IMV is a well known risk factor for the development of bronchopulmonary dysplasia (BPD) - a condition associated with significant morbidity and mortality $[10,11]$.

To minimize the need of IMV, various noninvasive respiratory support modalities are available in neonatal intensive care units (NICU). A systematic review has shown that non-invasive positive pressure ventilation (NIPPV) reduces the need for IMV (within 1 week from extubation) more effectively than CPAP, although it is not clear if NIPPV may impact on the longterm need for ventilation, $\mathrm{BPD}$ or mortality [12]. The main drawback of neonatal NIPPV is the lack of synchronization, which is difficult to achieve and is often unavailable. A more recent alternative technique is noninvasive high frequency oscillatory ventilation (NHFOV) which consists on the application of a bias flow generating a continuous distending pressure with oscillations superimposed on spontaneous tidal breathing with no need for synchronization. The physiological, biological and clinical characteristics of NHFOV have been described elsewhere [13].

To date, there are few data about the use of NHFOV after extubation in preterm infants [14]. Relatively small case series or retrospective cohort studies suggested safety, feasibility and possible usefulness of NHFOV and have been reviewed elsewhere [13]. The only randomized parallel clinical trial comparing NHFOV to biphasic CPAP, in babies failing CPAP [15], has been criticized for methodological flaws and for not taking respiratory physiology into account [16]. An European survey showed that, despite the absence of large randomized clinical trials, NHFOV is quite widely used and no major side effects are reported, although large data about NHFOV safety are lacking [17]. This may be due to the relative NHFOV easiness of use but evidence- and physiology-based data are warranted about this technique.

\section{Need for a physiology-driven trial}

The noninvasive respiratory support policy may fail for several reasons such as, for instance, apneas, upper airways obstruction, technical malfunctioning or increasing work of breathing due to worsening of parenchymal lung disorder. NHFOV might be beneficial in this latter case, that is in neonates needing lung recruitment with high distending pressure to open their lungs. This may be the case of extremely preterm, BPD-developing neonates who have increased airway resistances, while they are subjected to a deranged alveolarization and lung growth. More in general, neonates presenting with respiratory acidosis may also benefit from NHFOV.

NHFOV may be beneficial because it allows to increase mean airway pressure (Paw) avoiding gas trapping and hypercarbia, thanks to the superimposed high frequency oscillations. NHFOV also provides the advantages of invasive high frequency oscillatory ventilation (no need for synchronization, high efficiency in $\mathrm{CO}_{2}$ removal, less volume/barotrauma) and nasal CPAP (noninvasive interface, oxygenation improvement by the increase in functional residual capacity through alveolar recruitment). Several animal and bench studies investigated the physiology and peculiarities of NHFOV [13] and these data should be used to conduct a physiology- 
guided trial in order to avoid errors done in the old trials about invasive high frequency ventilation [16].

This study will be the first large trial aiming to compare the long-term use of CPAP vs NIPPV vs NHFOV in preterm neonates after surfactant replacement and during their entire NICU stay, to reduce the total need/ duration of IMV. Noninvasive respiratory support is often used for several weeks, especially in extremely preterm neonates, but there are no clear data about the long-term effect of the different respiratory modalities and ours will be the first study in investigating this issue. Since there is a lack of formal data regarding NHFOV safety, some safety outcomes will also be addressed. Specific subgroup analyses will be conducted for prespecified groups of patients who are more likely to benefit from NHFOV, according to the above-described physiological characteristics. We hypothesized that NHFOV is more efficacious than CPAP or NIPPV to reduce the need for IMV in neonates born between 25 and 32 weeks' gestation, after their first extubation and until their final NICU discharge.

\section{Methods and design}

\section{Trial design}

This will be an assessor-blinded, multi-center, threearms, parallel, randomized, controlled trial with a superiority design, conducted in Chinese NICUs. Since safety data will also be analyzed, it may be considered the equivalent of a phase II/III trial. Since the trial will enroll all eligible patients irrespective of their lung mechanics/ physiopathology and the eligibility will be judged on the basis of simple clinical data commonly used in daily NICU care, it may be defined a pragmatic trial [18]. In fact, no particular entry criteria or diagnostic procedure will be required to enroll patients; no biological samples will be collected and no additional measures will be taken. Conversely, since subgroup analyses will be performed on patients defined a posteriori according to their actual lung physiopathology, they should be considered explanatory subgroup (preliminary) analyses [18]. Results of subgroup analyses will anyway need confirmation in future, specifically designed trials. A total of 69 NICUs are included in this trial (Fig. 1). All these NICUs belong to 30 provinces or cities or autonomous regions of Chinese mainland (apart from Tibet which has been excluded for the high altitude). The trial has been designed with the collaboration of an European investigator expert in NHFOV (DLD) and will have an international expert panel performing the interim analysis.

\section{Study aim and hypothesis}

Our aim is to verify the hypothesis that NHFOV is more efficacious than CPAP or NIPPV to reduce the need for IMV in neonates born between 25 and 32 weeks' gestation, after their first extubation and until their final NICU discharge.

\section{Inclusion criteria}

For a neonate to be included 4 criteria must be fulfilled: (1) gestational age (GA) between $25^{+0}$ and $32^{+6}$ weeks (estimated on the postmenstrual date and early gestation ultrasonographic findings); (2) assisted with any type of endotracheal ventilation; (3) post-conceptional age $<36$ weeks; (4) ready to be extubated for the first time (extubation readiness requires all the following criteria: a.

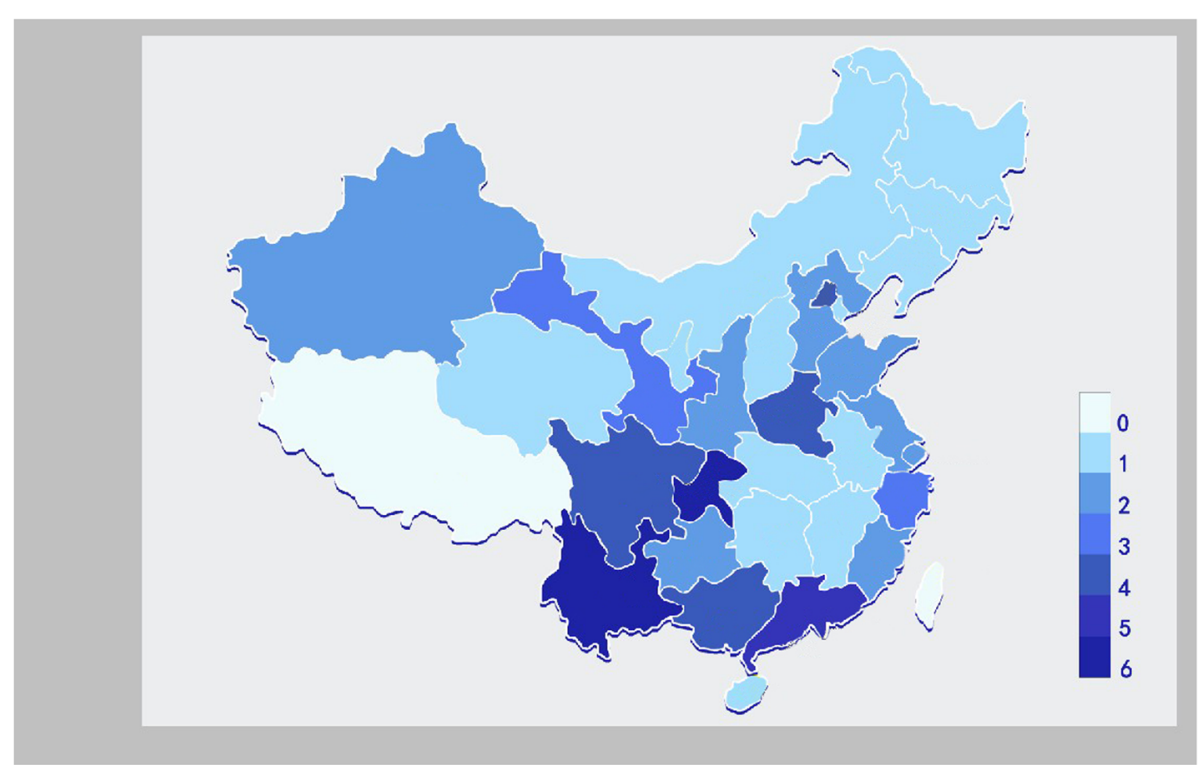

Fig. 1 Neonatal Intensive Care Units participating to the trial. Different colors represent the number of NICU participating in each area 
having received at least one loading dose of $20 \mathrm{mg} / \mathrm{kg}$ and $5 \mathrm{mg} / \mathrm{kg}$ daily maintenance dose of caffeine citrate; b. $\mathrm{pH}>7.20$ and $\mathrm{PaCO}_{2} \leq 60 \mathrm{mmHg}$ (these may be evaluated by arterialized capillary blood gas analysis or appropriately calibrated transcutaneous monitors [19] see Appendix. Venous blood gas analysis cannot be used); c. Paw of 7-8 or 8-9 $\mathrm{cmH}_{2} \mathrm{O}$, in conventional and oscillatory ventilation, respectively [6]; d. $\mathrm{FiO}_{2} \leq$ 0.30 ; e. sufficient spontaneous breathing effort, as per clinical evaluation [20]).

\section{Exclusion criteria}

Neonates who never needed intubation and IMV are not eligible for the study; similarly, a neonate randomized but never extubated is not eligible in the study. Moreover, neonates with at least one of the following criteria are also not eligible: (1) major congenital anomalies or chromosomal abnormalities; (2) neuromuscular diseases; (3) upper respiratory tract abnormalities; (4) need for surgery known before the first extubation; (5) Grade IVintraventricular hemorrhage (IVH) [21] occurring before the first extubation; (6) birth weight $<600 \mathrm{~g}$; (7) suspected congenital lung diseases (such as genetic anomalies of surfactant metabolism) or malformations (such as cystic adenomatous malformations, sequestration, diaphragmatic hernia) or pulmonary hypoplasia. More details are available in the appendix.

\section{Randomization}

Neonates will be randomized and assigned either to CPAP, NIPPV or NHFOV arms with a 1:1:1 ratio, when patients fulfil all inclusion criteria and extubation is deemed imminent (anyway within $1 \mathrm{~h}$ ). Randomization cannot be done earlier. Simple randomization will be done according to a computer-generated random number table and will be posted in a specific secured website 24/7 available. Twins will be allocated in the same treatment group. Infants randomized to one arm cannot crossover to the other or vice-versa during the study. Patients will remain under the assigned respiratory support until the weaning criteria will be met (see below). In case of intubation, when the baby will be extubated, he will receive again his original treatment according to randomization. This is summarized by Fig. 2 .

\section{Blinding}

Blinding towards the caregivers is impossible and blinding towards the patients makes no sense. However, outcomes' assessors will be blinded, as endpoints will be recorded by assessors not involved in patients' care: they will review patients' files masked for the type of treatment. An assessor per each participating NICU will be nominated. Moreover, investigators performing the final statistical analyses will also be blinded to the treatment allocation.

\section{Primary outcomes}

The primary outcomes will be: (1) duration of IMV (in days) from the randomization; (2) ventilator-free days (calculated as described in the appendix); (3) the number of reintubation. Neonates will be re-intubated if one of the following occurs:

a. severe respiratory acidosis (defined as $\mathrm{PaCO}_{2}>65$ $\mathrm{mmHg}$ with $\mathrm{pH}<7.2$ );

b. hypoxia refractory to study intervention (defined as need for $\mathrm{FiO}_{2}>0.4$ to obtain $\mathrm{SpO}_{2}<90 \%$, with and maximal pressures allowed in the study arm - see below) for at least $4 \mathrm{~h}$;

c. severe apnea (defined as recurrent apnea with $>3$ episodes/h associated with heart rate $<100 /$ min or a single episode of apnea requiring bag and mask ventilation, or associated with $\mathrm{SpO}_{2}<85 \%$ and $\mathrm{FiO}_{2}>0.6$ );

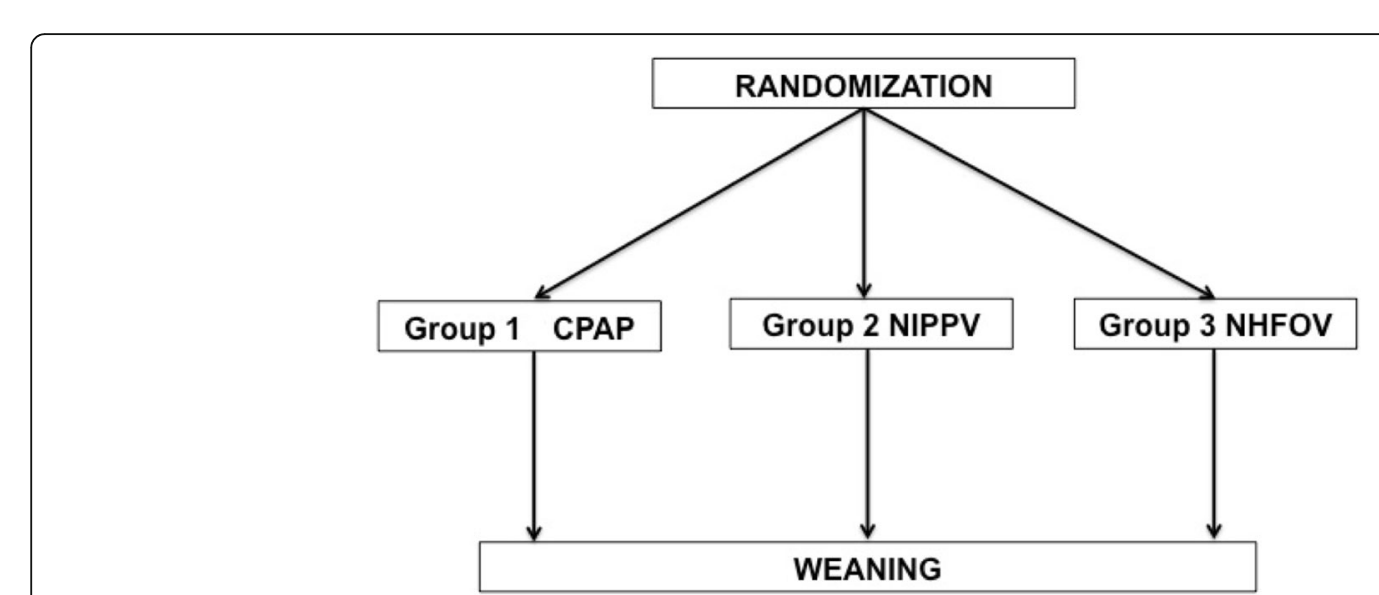

Fig. 2 Basic study design. Neonates will stay on the assigned intervention until the final weaning. No cross-over allowed. In case of intubation, when the baby will be extubated, he will receive again his original treatment according to randomization 
d. pulmonary hemorrhage (defined as brightly blood tracheal secretion associated with sharp increase in oxygen and Paw and with the occurrence of "white lungs", new infiltrates or consolidations at the chest $\mathrm{X}$-rays or lung ultrasound);

e. severe respiratory distress (defined as Silverman score $>4$ ) for at least $4 \mathrm{~h}$;

f. hemodynamic instability, defined as mean arterial pressure $<10$ th percentile of appropriate nomograms $[22,23]$ or anyway need of dopamine (if $>5 \mathrm{mcg} / \mathrm{Kg} / \mathrm{min}$ ) or dobutamine (if $>5 \mathrm{mcg} / \mathrm{Kg} /$ min) or any dose of noradrenaline, adrenaline, milrinone, nitric oxide or other pulmonary vasodilators.

g. cardio-respiratory arrest.

\section{Secondary outcomes}

The secondary outcomes will be: (1) airleaks (pneumothorax and/or pneumomediastinum) occurred after the extubation; (2) BPD, defined according to the NICHD definition [24]; (3) hemodynamically significant patent ductus arteriosus (PDA), defined according to local NICU protocols; (4) retinopathy of prematurity (ROP) > 2nd stage [25]; (5) necrotizing enterocolitis (NEC) $\geq 2$ nd stage [26]; (6) IVH > 2nd grade [21]; (7) need for postnatal steroids; (8) in-hospital mortality; (9) composite mortality/BPD; (10) weekly weight gain (in grams/d) for the first 4 weeks of life or until NICU discharge, whichever comes first.

\section{Safety outcomes}

The safety outcomes will be the following: (1) weekly number of vomiting/d; (2) weekly volume of gastric residual (ml/d); (3) weekly number of apneas/d; (4) nasal skin injury (weekly defined by a clinical score [27] as: 0 (zero, absence of injury), stage I (non-blanching erythema), stage II (superficial erosion), stage III (necrosis of full thickness of skin) - more details in the appendix). These outcomes will be averaged over each week for the first 4 weeks of life or until NICU discharge, whichever comes first. Finally, (5) Premature Infant Pain Profile (PIPP) score [28] will be considered (averaged from values available in the first $48 \mathrm{~h}$ from the allocation to CPAP, NIPPV or NHFOV). Investigators will also record any serious adverse event (defined as an untoward medical occurrence that is believed by the investigators to be causally related to study-intervention and results in any of the following: life-threatening condition (that is, immediate risk of death); severe or permanent disability, prolonged hospitalization).

\section{Study intervention}

When the neonate had fulfilled the extubation criteria, this latter will take place with a gentle intratracheal suction, following local clinical policies. Upper airways will then be suctioned and intervention will be started immediately as follows:

\section{Ventilators}

- CPAP: CPAP will be provided by either variable flow or continuous flow devices, as there is no clear evidence that one type of CPAP generator would be better than any other [29].

- NIPPV: NIPPV will be provided by any type of neonatal ventilator able to generate enough pressure according to the protocol (see below).

Synchronization will not be applied, as many currently marketed neonatal ventilators do not provide it for NIPPV [30].

- NHFOV: NHFOV will only be provided with piston/ membrane oscillators able to provide an active expiratory phase (that is, Acutronic FABIAN-III, SLE 5000, Loweinstein Med LEONI+, Sensormedics 3100A). Other machines pretending to provide high frequency ventilation using other technologies will not be used.

Before the beginning of the study all ventilators will be checked to ensure that there is no malfunction.

\section{Interfaces}

CPAP, NIPPV and NHFOV will be administered through short, low-resistance binasal prongs and/or nasal masks, since these are supposed to be the best in terms of resistive charge and leaks $[4,5]$. Nasal prongs size will be chosen according to the nares' diameter as the best fitting ones (the largest ones fitting the nares without blanching the surrounding tissues) and following manufacturer's recommendations. Nasal masks will also be appropriately sized according to manufacturer's recommendations. Alternating masks and prongs, according to clinical evaluation, is allowed in order to reduce the risk for nasal skin injury. Particular care (e.g.: pacifiers, positioning, nursing) will be applied to reduce leaks and improve patients' comfort. These latters will be evaluated through a dedicated $30^{\prime}$ observation period when study the intervention will be instigated. Non-pharmacological sedation with pacifiers and $33 \%$ glucose solution will be provided, when needed; no other sedation will be allowed. RAMCannula ${ }^{\circ}$ are not allowed in the trial due to their resistive charge and their relevant pressure leaks $[31,32]$.

\section{Ventilatory management}

The three interventions will be managed as follows:

- CPAP: Neonates assigned to the CPAP group were initiated on a pressure of $5 \mathrm{cmH}_{2} \mathrm{O}$. CPAP can be 
raised in steps of $1 \mathrm{cmH}_{2} \mathrm{O}$ up to $8 \mathrm{cmH}_{2} \mathrm{O}$. If this is not enough to maintain $\mathrm{SpO}_{2}$ between 90 and $95 \%$, $\mathrm{FiO}_{2}$ will be increased up to 0.40 .

- NIPPV: neonates assigned to the NIPPV group will be started with the following parameters: a) positive end-expiratory pressure (PEEP) of $4 \mathrm{cmH}_{2} \mathrm{O}$ (can be raised in steps of $1 \mathrm{cmH}_{2} \mathrm{O}$ to $\max 8 \mathrm{cmH}_{2} \mathrm{O}$, according to the oxygenation). b) Peak Inspiratory Pressure (PIP) of $15 \mathrm{cmH}_{2} \mathrm{O}$ (can be raised in steps of $1 \mathrm{cmH}_{2} \mathrm{O}$ to max $25 \mathrm{cmH}_{2} \mathrm{O}$, according to oxygenation, $\mathrm{PaCO}_{2}$ levels and the chest expansion); maximal allowed $\mathrm{FiO}_{2}$ will be 0.40 and $\mathrm{SpO}_{2}$ targets will be $90-95 \%$. c) inspiratory time (IT) will be $0.45-0.5 \mathrm{~s}$ (according to clinicians' evaluation of leaks and the appearance of the pressure curve: a small pressure plateau is required and flow may be set accordingly) and rate will be started at $30 \mathrm{bpm}$ (can be raised in steps of $5 \mathrm{bpm}$ to max $50 \mathrm{bpm}$, according to $\mathrm{PaCO}_{2}$ levels).

- NHFOV: neonates assigned to NHFOV will be started with the following boundaries, according to available physiological and mechanical data, as suggested elsewhere [13]: a) Paw of $10 \mathrm{cmH}_{2} \mathrm{O}$ (can be changed in steps of $1 \mathrm{cmH}_{2} \mathrm{O}$ within the range range 5-16 $\mathrm{cmH}_{2} \mathrm{O}$ ); Paw will be titrated (within the range) according to open lung strategy, performing alveolar recruitment, similar to what is done in invasive high frequency oscillatory ventilation targeting a $\mathrm{FiO}_{2} \leq 25-30 \%$, as published elsewhere [33]. Maximal allowed $\mathrm{FiO}_{2}$ will be 0.40 and $\mathrm{SpO}_{2}$ targets will be $90-95 \%$. b) frequency of $10 \mathrm{~Hz}$ (can be changed in steps of $1 \mathrm{~Hz}$ within the range 8-15 $\mathrm{Hz}$ ). c) Inspiratory time $50 \%$ (1:1) [34]. d) amplitude $25 \mathrm{cmH}_{2} \mathrm{O}$ (can be changed in steps of $5 \mathrm{cmH}_{2} \mathrm{O}$ within the range 25-50 $\mathrm{cmH}_{2} \mathrm{O}$ ) [34, 35]; amplitude will be titrated according to $\mathrm{PaCO}_{2}$. It is not strictly necessary to have visible chest oscillations, as $\mathrm{PaCO}_{2}$ elimination during NHFOV also occurs in the upper airway dead space [36]. In case of hypercarbia, amplitude will be increased first and then frequency will be lowered (within the above-described ranges), however, if nasal masks are used, the amplitude should be kept at the maximum and $\mathrm{PaCO}_{2}$ controlled by frequency titration, as oscillation amplitude using masks is more dampened [37, 38].

\section{Monitoring and concurrent treatments/diagnostic measures}

$\mathrm{PaCO}_{2}$ will be measured using arterialized capillary blood gas analysis and/or transcutaneous monitoring according to local policies. Transcutaneous monitoring will be performed according to the American Association of Respiratory Care guidelines [19] and the manufacturer's recommendations. Frequency of blood gas analysis will be decided by the attending clinicians. All neonates will be continuously monitored for $\mathrm{SpO}_{2}$, ECG, heart and respiratory rate. To avoid abdominal distention, a feeding tube will be placed in the stomach through the mouth and gas will be periodically aspirated according to nurses' evaluation in all study arms. Moreover, the following treatments or tests will be provided:

- Heart ultrasound to evaluate cardiac morphology, pulmonary pressures and PDA, within the first $3 \mathrm{~d}$ of life and subsequently repeated, if needed.

- Cerebral ultrasound within $48 \mathrm{~h}$ of life and weekly thereafter, until discharge, if needed.

- Routine measures to prevent BPD; routine fluid/ nutritional policy; routine caffeine therapy.

- Placement of umbilical central venous catheter and/ or peripherally inserted central venous lines. Placement of arterial lines if needed, according to local policies.

- Routine therapies according to local policies (i.e.: antibiotics, PDA closure drugs...).

In general, routine medical care and nursing will not be changed because of the study, out of the trial intervention; the clinical care will be identical in the three study arms. No additional blood samples are required solely for study purposes.

\section{Weaning from study interventions}

The study intervention will be progressively weaned, according to clinical evaluation and respecting the following guidelines:

in the CPAP arm, pressure will be reduced by 1 $\mathrm{cmH}_{2} \mathrm{O}$ steps down to a minimum of $3 \mathrm{cmH}_{2} \mathrm{O}$; in the NIPPV arm, PIP and PEEP will be reduced by $1 \mathrm{cmH}_{2} \mathrm{O}$ steps down to a minimum of 5 and 3 $\mathrm{cmH}_{2} \mathrm{O}$, respectively. Similarly, frequency will be reduced to a minimum level of $20 \mathrm{bpm}$ in steps of 5 bpm.

in the NHFOV arm, amplitude will be reduced to the minimum initial level of $20 \mathrm{cmH}_{2} \mathrm{O}$ and Paw will be reduced by $1 \mathrm{cmH}_{2} \mathrm{O}$ down to a minimum of 3-5 $\mathrm{cmH}_{2} \mathrm{O}$ (depending on the ventilator used).

The study intervention (CPAP, NIPPV or NHFOV) will be stopped when the above-described minimum parameters are reached and maintained for at least $48 \mathrm{~h}$ with the following: (1) $\mathrm{FiO}_{2} \leq 0.25$; (2) Silverman score < 3; (3) no apneas or bradycardia without spontaneous recovery. If a baby will desaturate $\left(\mathrm{SpO}_{2}<85 \%\right.$ with $\mathrm{FiO}_{2}>$ $25 \%$ ) or has relevant dyspnea (Silverman $\geqq 3$ ) or more than 3 apneas/d, the intervention (CPAP, NIPPV or NHFOV) will be restarted for at least $48 \mathrm{~h}$ and then re- 
evaluated. The end of study intervention may occur at any time during hospitalization if the above described criteria are met. When study interventions end, the neonate may be placed under low flow oxygen therapy (max $1 \mathrm{~L} / \mathrm{min}$ ), if needed, according to clinicians' evaluation and local protocols. Anyway, when a post-conceptional age of 36 weeks is reached, if the patient still needs noninvasive respiratory support, he/she will be shifted to CPAP and managed according to clinical evaluation and local policies.

\section{End of the study}

A patient may exit from the study for any of the following reasons:

1. Death

2. In any case, when the 36 weeks' post-conceptional age is reached.

3. If parents or guardians withdraw an already given consent for the participation (in that case the patient will keep receiving the whole routine clinical care; data acquired will be immediately destroyed).

\section{Training}

Since not all clinicians are well versed in all respiratory techniques and, particularly, NHFOV is a relatively new technique, the training is capital for the trial success. The protocol will be diffused between participating centers at least 3 months before the study begins. One investigator (YS) will explain the study protocol in an in-person meeting with all investigators. During the 3 months, clinicians will familiarize with the protocol and the respiratory techniques and an expert in NHFOV (DLD) will be available to solve any doubt. A dedicated social media chat has been set to facilitate these contacts. Moreover, an expert in NHFOV (DLD) will conduct, site visits and a webinar teaching about the technique and will personally visit some participating NICUs.

\section{Sample size calculation}

It is difficult to calculate a sample size, since this is the first trial to investigate CPAP vs NIPPV vs NHFOV in post-extubation phase in preterm babies. However, a previous prospective, cohort, non-randomized, pilot study comparing post-extubation NIPPV and NHFOV in preterm neonates provided data about the primary outcome "duration of mechanical ventilation". This preliminary study showed a reduction of $\approx 30 \%$ for babies receiving NHFOV, as compared to those treated with NIPPV, but it has been presented only as abstract so far [39]. A randomized trial of NIPPV vs CPAP by Ramanathan et al. showed a similar reduction [40]. Since these trials have not the same design of ours, we decide to be more prudent and we aimed a difference of $20 \%$ in the duration of mechanical ventilation. Considering an alpha-error of 0.05 (with a Bonferroni correction at 0.017) and a power of 95\%, 480 neonates should be enrolled in each arm (with a 1:1:1 design). Thus, a total of at least 1440 neonates will be enrolled. Sample size calculation has been performed with GPower3.1.9.3 [41]. We do not foresee any problem in reaching this sample size over 12-18 months, given the large network and the number of potentially eligible neonates.

\section{Data collection}

All data can be obtained from the clinical notes. Data will be recorded in real time on web-based case report forms provided by OpenCDMS. The website will be tested with fictitious data before the actual enrolment. Data will be entered by an assessor per each center. Assessors will be research nurses or local investigators blinded to the study intervention and not directly involved in patients' care. Access to the form will be secured and patients will be de-identified. Clinical information will be collected at the following time-points:

1. Before the intervention begins: information on eligibility; baseline clinical informations, respiratory diagnosis, critical risk index for babies-II (CRIB-II) score [41].

2. Following study intervention: ventilatory parameters, $\mathrm{SpO}_{2}$, blood gas values before the extubation if available. $\mathrm{PaO}_{2}, \mathrm{PaCO}_{2}, \mathrm{SpO}_{2}$ and $\mathrm{pH}$ between $6 \mathrm{~h}$ and $24 \mathrm{~h}$ from the extubation.

3. Follow-up: NICU length stay, duration of IMV, number of reintubation, ventilator free days, duration of oxygen therapy, duration of the study intervention (CPAP, NIPPV or NHFOV), airleaks, PDA, BPD, ROP $>2$ nd stage, NEC $\geq 2$ nd stage, IVH $>$ 2nd grade, need for postnatal steroids, inhospital mortality, composite mortality/BPD, weekly weight gain (in grams/d) for the first 4 weeks of life or until NICU discharge, whichever comes first. Moreover, the following safety data will be recorded: weekly number of vomiting/d, weekly volume of gastric residual $(\mathrm{ml} / \mathrm{d})$; weekly number of apneas/d; nasal skin injury (weekly defined by a 12-3 clinical score [27]). These outcomes will be averaged over each week for the first 4 weeks of life or until NICU discharge, whichever comes first. Finally, PIPP score [28] will be recorded in the first $48 \mathrm{~h}$ from the allocation. Abdominal circumference at $48 \mathrm{~h}$ and $96 \mathrm{~h}$ from the instigation of CPAP, NIPPV or NHFOV will also be recorded. 


\section{Statistics}

Data analysis will be performed blindly to the type of treatment received. An intention-to-treat analysis will be applied. An interim analysis will be performed at approximately $50 \%$ of the enrolment together by international expert panel. First, data will be checked for normality using Kolmogorov-Smirnov test and results will be presented as odds ratio (OR) and 95\% confidence interval $(\mathrm{CI})$ or adjusted $\mathrm{OR}$ and $95 \% \mathrm{CI}$, and mean \pm standard deviation or median [quartiles], as appropriate.

Univariate logistic or linear regressions will be performed, according to the type of variable, as appropriate. Univariate Cox's proportional regression will be used for mortality and the duration of IMV. Multivariate regressions will also be performed for selected outcomes, if needed (that is, if a baseline characteristic differs between the two arms with a $p<0.2$ at the univariate analysis the results will be adjusted for that variable). For each multivariate analysis, multicollinearity will be previously checked considering condition index of Eigenvalues and Variance Inflation Factor [43, 44]. $p$ values $<0.05$ will be considered statistically significant.

The following sub-group analyses will be performed:

1. Analysis for babies $\leq 28$ weeks' gestation.

2. Analysis for babies who have been invasively ventilated for at least 1 week from birth.

3. Analysis for babies with $\mathrm{PaCO}_{2}>50 \mathrm{mmHg}$ before the extubation or at the $6 \mathrm{~h}$ or $24 \mathrm{~h}$ after extubation.

These subgroup analyses have been chosen trying to give more insight about the use of the trialed respiratory techniques for patients with different respiratory physiopathology, as there is no doubt that a more preterm baby have a higher risk of reintubation or that those stacked on the ventilator have "evolving BPD". However, subgroups have been selected using simple clinical data to remain pragmatic and facilitate the recruitment.

\section{International colleagues' panel}

This panel will analyze all data in an interim analysis at approximately $50 \%$ of the trial enrolment. The panel will be composed by international neonatologists or pediatric intensivists experts in respiratory care. The risk for patients' safety is estimated as very low, since the interventions are known and already used in NICU care. The panel will analyze all data outcome, both on efficacy and safety. This is unusual in a trial about neonatal ventilation but will help to increase the quality of data. Unless this happens, however, the investigators will remain ignorant of the interim results. The board will advice the principal investigator (YS) who will remain the only responsible for the trial conduction and for any eventual decision to stop or continue it. The board will also give some advices on quality of data and their analysis: the principal investigator will remain responsible for the final data, analysis and study results. The board members will be completely independent from the trial, not working at any institution enrolling patients and with no direct conflict of interest.

\section{Discussion}

\section{On the trial methods and limitations}

Ours is a study trying to apply the methodology of pharmacological trials to neonatal mechanical ventilation, a field where it is often difficult to do so for practical reasons (blinding difficulties, long study times, difficult recruitment, lack of funding). Nonetheless, ours is also a "physiological" trial, since interventions are applied based on physiological perspective, in order to optimize the use of the different ventilatory techniques. This has been often neglected in the past, but we are exploiting the previous bench and in vivo mechanical data [13] in order to optimize the use of NHFOV. Therefore, we hope to provide high quality data, although further trials may be required, as this is only the first large one on NHFOV and some questions will remain unanswered (see below).

Moreover, we applied a pragmatic design in order to increase generalizability of our results [18]. This means enrolling many babies in the common daily NICU care, irrespective of their actual mechanical and biological lung condition, without any particular test prior to enrolment. However, an explanatory design may be more appropriate for a recently introduced new therapeutic intervention [44, 45], since a pragmatic design may lead to dilute the effect of the interventions and mask their effect on a particular type of patients. This is the reason why we also previewed three subgroup analyses according to predefined criteria in order to identify patients (a posteriori) for some of their characteristics. This has been possible because the large population size will allow us to recruit a certain number of patients with homogeneous traits and try to have some advantages of the explanatory design in the subgroup analyses [18]. However, results of these subgroup analyses, even if promising, must be considered preliminary and will unavoidably require further confirmation in subsequent specifically designed trials. Alternatively, future trials using more stringent criteria, and/or also using less common monitoring techniques, will be useful to better describe lung physiopathology and recruit patients with a fully explanatory design.

We cannot clarify if increasing CPAP levels may eventually approach results obtained with NIPPV or NHFOV used with higher mean airway pressure. This could be 
considered as a trial limitation, albeit we must consider that:

- the use of higher CPAP levels is controversial and not standardized. An open lung strategy during CPAP has never been formally studied while this has been done in invasive HFOV [33]. European guidelines do not advice to use higher CPAP levels $[6,46]$. Anyhow, we could not change this point, as higher CPAP levels are not common standard of care in Chinese NICU and the trial needed to respect this in order to be pragmatic.

- Increasing CPAP level without any real ventilation, may increase the risk of gas trapping (even if this may not be clinically evident as airleak syndrome). The risks or consequences related to this might not be accurately detected in a study on non-invasive respiratory support where patients are not closely monitored as those intubated or those enrolled in explanatory trials using particular techniques looking at lung volume (for instance, electrical impedance tomography [47], respiratory inductance pletysmography [48], or semi-quantititative lung ultrasound [49]).

- The presence of oscillatory pressure waveform will have an effect only on $\mathrm{PaCO}_{2}$ levels, as previously by demonstrated by basic physiology $[13,36]$ and metaanalysis of NHFOV clinical trials published so far [50]. This is the reason why we included a subgroup analysis for hypercarbic babies in order to detect as much as possible the potential effect of this ventilatory trait.

- If allowing higher mean airway pressures in NHFOV than in CPAP may influence the interpretations of results, this is also true for NIPPV, which allows increasing mean airway pressures, while providing a real ventilation by using a conventional volume delivery. We believe that this may be a main reason behind the lack of diffusion and standardization of higher levels CPAP policies, whereas techniques actually providing a true ventilation are felt safer from a physiopathological point of view.

Thus, we are interesting and willing to see if NHFOV is able to provide advantages on CPAP (used with common pressure levels), due to its global technical characteristics, including the possibility to increase constant distending pressure preventing the development of airleaks with the application of the oscillatory pressure waveform.

Finally, we do not allow any cross-over between the study arms for the following reasons. While there are some data indicating possible efficacy of NIPPV to avoid re-intubation, this evidence is generally considered of moderate-low quality [12]. Moreover, the general absence of synchronization is reducing the efficacy and creating an unjustified difference with NIPPV provided in critically ill children and adults. As such, European guidelines do not clearly suggest the use of NIPPV, unless it is synchronized [46], and there is no clear guidliens on this issue from the American Academy of Pediatrics. Therefore, we decided not to allow the crossover and choose directly to the best evidenced and more widely used rescue practice which remains endotracheal intubation and IMV. This is also in agreement with the pragmatic study design.

\section{Ethical considerations}

This trial is worth to be conducted given the uncertainty about the superiority of one respiratory technique over the others, especially for babies at highest risk. Moreover, NHFOV might be actually superior to the other techniques, as we may hypothesize this from the currently available data [13]. NHFOV has been already studied in preliminary cross-over trials, in bench and animal studies [13], while invasive HFOV is often used for severe respiratory failure. NIPPV has been studied in several randomized controlled trials enrolling smaller population and/or without triple comparison against CPAP and NHFOV [12]. Thus, the tested interventions are not totally new and there is a great drive towards noninvasive ventilation in NICU care: this study is a new step within this framework.

Thus, the risks for babies are minimized and the monitoring will quickly report any possible problem. Out of the studied intervention, the participation to the study will not change the routine clinical assistance. Data will be anonymously recorded, secured and accessible only to the investigators and to the parents/guardians. In no case the recorded data will be used for purposes out of those specified in the trial protocol. Moreover, the trial is only funded by a public Chinese research program, thus it will not have external industrial influences and has the merit to try filling the lack of public funding for neonatal ventilation trials [51].

\section{Publication policy}

Study results will be presented to each investigator by teleconference and/or e-mail by the principal investigator (YS). If possible an investigator meeting in occasion of one of the major congresses in the field of pediatrics or critical care (the European Society for Pediatric Research or European Society for Pediatric and Neonatal Intensive Care Congresses or the Pediatric Academic Societies Meeting) will be organized. Data will be also partially presented at these meetings. Abstracts and manuscripts will be circulated between all investigators for revision and will be approved by all authors in their 
final version. All manuscripts will be authored by a group authorship: full results will be published in a major journal in the field of general medicine or pediatrics or critical care. Authorship criteria of the International Committee of Medical Journal Editors will be followed.

\section{Appendix}

\section{Clarifications for exclusion criteria}

Neonates who never needed intubation and IMV are not eligible for the study; similarly, a neonate randomized but never extubated is not eligible. This means that, if a randomized neonate is not actually extubated within $1 \mathrm{~h}$, because there has been a worsening of his conditions or death, he is excluded from the study. Randomization must be done as close as possible to the extubation, once all inclusion criteria are fulfilled (see above), and anyway within $1 \mathrm{~h}$ from the actual extubation.

Some exclusion criteria are represented by congenital disorders. When a patient is affected by these disorders his biology and physiology are significantly deranged: they are not eligible and will not be randomized. If the condition has been discovered/suspected after the randomization but before the study inclusion (that is, before extubation), they will not receive the study intervention and will not entry in the study. If one of these conditions is diagnosed after the inclusion in the study, the neonate will be excluded a posteriori. This is the case of neonates with major congenital anomalies, chromosomal abnormalities, neuromuscular diseases, congenital upper respiratory tract abnormalities and congenital lung diseases or malformations or hypoplasia. Examples of these conditions are: genetic syndromes, surfactant protein deficiency, congenital adenomatous pulmonary malformations, congenital diaphragmatic hernia or sequestration, congenital hypoventilation syndrome, pulmonary hypoplasia or any metabolic disease. Same applies for the need for surgery anticipated antenatally or before the first extubation, as this is usually related to congenital malformations. These neonates are not eligible and will not be randomized. If the condition has been discovered/suspected after the randomization but before the study inclusion (that is, before extubation) they will not receive the study intervention and will not entry in the study. If a surgery will be needed later during the NICU hospitalization for other reasons (for instance for PDA ligation or NEC), the patient will regularly continue the trial. These needing surgery conditions will be noticed amongst the outcomes.

Grade IV-IVH known before the first extubation is a significant risk factor for prognosis and for quality of life. Continuing the NICU care in this situation may be considered unethical, depending to different local settings, cultures, ethical and religious beliefs. This may significantly impact on the trial outcomes. These neonates are not eligible and will not be randomized. If grade IV-IVH has been discovered/suspected after the randomization but before the study inclusion (that is, before extubation), they will not receive the study intervention and will not entry in the study. If Grade IV-IVH will be diagnosed after the study inclusion, the patient will continue the study regularly and this will be noticed amongst the outcomes.

\section{List of study definitions/assessments (in alphabetical order) \\ Antenatal steroids}

Antenatal steroid prophylaxis will be considered complete if two $12 \mathrm{mg}$-doses of betamethasone $24 \mathrm{~h}$ apart and between 1 day and 7 days before the delivery had been given.

\section{Blood gas analysis}

Blood gas values may only be obtained in following three ways (venous blood gas analysis is not allowed in the study).

- Arterial blood from indwelling arterial lines, if one of these was placed for clinical reasons. As these are likely to be unavailable in the majority of cases, the following two alternative techniques may be used. Blood gas analysis will be obtained upon attending neonatologist decision.

- Arterialized capillary blood gas analysis is performed, warming a patient's heel for $10^{\prime}$ and collecting $200 \mu \mathrm{L}$ of blood into a heparinized microtube. This must be analyzed by a blood gas analyzer within $5^{\prime}$. Blood gas analysis will be obtained upon attending neonatologist decision.

- Transcutaneous blood gas monitoring will be performed according to American Associations of Respiratory Care guidelines [19] and the device manufacturer's recommendations and using an electrode temperature of $44^{\circ} \mathrm{C}$ for a short time (max 10-15 min). Particular care must be provided to avoid skin injury in extremely preterm neonates: in some cases, a temperature of $42^{\circ} \mathrm{C}$ may be more suitable. Transcutaneous gas measurements will be obtained upon attending neonatologist decision.

BPD definition [24] for neonates $\leq 32$ weeks' gestation, as described in Fig. 3.

Clinical Risk Index for Babies (CRIB-II) score [42]. This is an estimator of the clinical severity at the NICU admission. CRIB-II score considers 4 variables: birth weight, GA, base excess within the $1^{\text {st }} \mathrm{h}$ of life and temperature at the admission. An online tool will be used to calculate the score. 


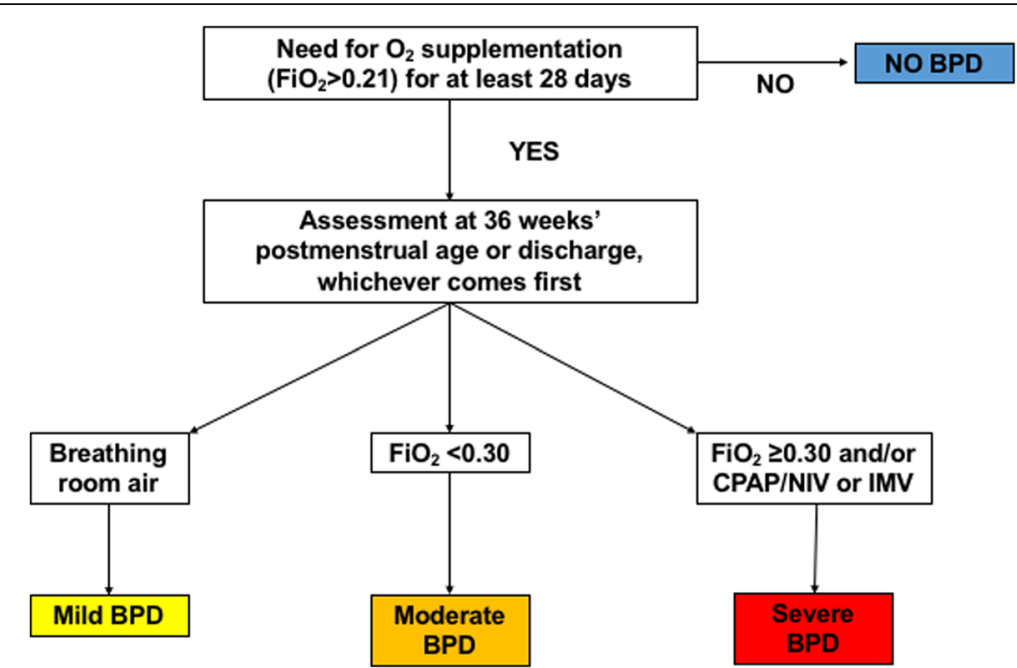

Fig. 3 Current bronchopulmonary dysplasia definition [24]. The figure represents the algorithms to apply the definition only in babies $\leq 32$ weeks' gestation, as these are those enrolled in the trial

Time on CPAP/NIPPV/NHFOV. Number of days spent under these respiratory supports will be registered and rounded to the closest entire number.

Gestational age (GA). GA is determined based on last menstrual period or early ultrasound scan (within the first trimester). If a discrepancy of more than 2 weeks exists, the early ultrasound scan will be chosen.

Nasal injuries. These are classified by using a clinical score [26] as stage I (non-blanching erythema), stage II (superficial erosion), stage III (necrosis of full thickness of skin) in the skin area in contact with nasal prongs, as described elsewhere [27]. The score will be 0 (zero), in case of absence of any injury.

Premature Infant Pain Profile (PIPP) score, as described elsewhere [28].

Pulmonary hypoplasia. This will be clinically defined if anamnestic (prenatal findings: small lung volume), imaging (diffuse chest $\mathrm{x}$-ray opacity or hypo-density) and clinical data (extremely low gestational age, olygoanhydramnios, severe pulmonary hypertension, refractory hypoxia) are present. Pulmonary hypoplasia usually does not allow survival.

Respiratory main diagnosis. A respiratory main diagnosis that required IMV ( \pm surfactant administration) has to be given according to the following criteria. RDS: respiratory distress appearing within the first $24 \mathrm{~h}$ of life, with complete, sustained, and prompt response to surfactant or lung recruitment or both; additional nonmandatory criteria are lung imaging (chest X-rays or ultrasound, according to local policies) supporting the diagnosis or lamellar body counts $\leq 30000 / \mathrm{mm}^{3}$, or both [52]. Pneumonia: broncho-alveolar lavage fluid or blood positive culture or C-reactive protein and/or procalcitonin beyond the normal values, together with radiological signs of infection(infiltrates and/or consolidation and/or loss of aeration) [53].

Sepsis (international pediatric sepsis definition): presence of systemic inflammatory response syndrome (SIRS) together with a suspected or proven (by positive culture, tissue stain, or polymerase chain reaction test) infection caused by any pathogen or a clinical syndrome associated with a high probability of infection [54]. Infection is suspected according to anamnesis, clinical exam, imaging or laboratory tests. Evidence of SIRS is given by the presence of at least two of the following four criteria, one of which must be abnormal temperature or leukocytes:

- Core temperature $>38.5^{\circ} \mathrm{C}$ or $<36^{\circ} \mathrm{C}$.

- Tachycardia, defined as a mean heart $>180 \mathrm{bpm}$ in the absence of external stimulus, chronic drugs, or painful stimuli; or otherwise unexplained persistent elevation over a 0.5 - to 4 -h time period OR bradycardia, defined as a mean heart rate $<100 \mathrm{bpm}$ in the absence of external vagal stimulus, -blocker drugs, or congenital heart disease; or otherwise unexplained persistent depression over a 0.5 -h time period.

- Mean respiratory rate $>60 / \mathrm{min}$ or need for IMV for an acute process not related to underlying neuromuscular disease or the receipt of general anesthesia.

- Leukocyte count elevated or depressed or $10 \%$ immature neutrophils.

Meconium aspiration syndrome (MAS): Presence of meconium-stained amniotic fluid and secretions upon tracheal suctioning with onset of respiratory distress early from birth and chest X-rays or lung ultrasound 


\begin{tabular}{|c|c|c|c|c|c|c|}
\hline \multirow[b]{2}{*}{ TIMEPOINT } & \multirow{2}{*}{$\begin{array}{c}\text { Enrolment } \\
\qquad t_{1}\end{array}$} & \multirow{2}{*}{$\begin{array}{c}\text { Allocation } \\
0\end{array}$} & \multicolumn{3}{|c|}{ Post-allocation } & \multirow{2}{*}{$\begin{array}{c}\text { Close-out } \\
\text { NICU } \\
\text { discharge }\end{array}$} \\
\hline & & & $\begin{array}{c}1^{\text {st }} \\
\text { extubation }\end{array}$ & $\begin{array}{c}4 \text { weeks } \\
\text { after } 1^{\text {st }} \\
\text { extubation }\end{array}$ & $\begin{array}{l}\text { Weaning (or } \\
36 \text { wks post- } \\
\text { conceptional } \\
\text { age) } \\
\end{array}$ & \\
\hline \multicolumn{7}{|l|}{ ENROLMENT: } \\
\hline \multirow{2}{*}{$\begin{array}{l}\text { Eligibility screen } \\
\text { Informed consent }\end{array}$} & $x$ & & & & & \\
\hline & $x$ & & & & & \\
\hline Allocation & & $X$ & & & & \\
\hline \multicolumn{7}{|l|}{ INTERVENTIONS: } \\
\hline \multicolumn{7}{|l|}{ CPAP } \\
\hline \multicolumn{7}{|l|}{ NIPPV } \\
\hline \multicolumn{7}{|l|}{ NHFOV } \\
\hline \multicolumn{7}{|l|}{ ASSESSMENTS: } \\
\hline \multirow{3}{*}{$\begin{array}{r}\text { Duration of IMV } \\
\text { Ventilator free } \\
\text { days }\end{array}$} & & & & & & $x$ \\
\hline & & & & & & $x$ \\
\hline & & & & & & $x$ \\
\hline \multirow[t]{2}{*}{ Airleaks } & & & & & & $x$ \\
\hline & & & & & $x$ & \\
\hline$B P D$ & & & & & & $x$ \\
\hline$P D A$ & & & & & & $x$ \\
\hline ROP & & & & & & $x$ \\
\hline$N E C$ & & & & & & $x$ \\
\hline \multirow[t]{2}{*}{$\begin{array}{r}\text { Need for postnatal } \\
\text { steroids }\end{array}$} & & & & & & $x$ \\
\hline & & & $\mathrm{x}$ & $x$ & $x$ & $x$ \\
\hline $\begin{array}{r}\text { In-hospital motality } \\
\text { Weekly weight } \\
\text { gain }\end{array}$ & & & & $x$ & & $\mathrm{X}^{*}$ \\
\hline $\begin{array}{r}\text { Weekly n. of } \\
\text { vomiting }\end{array}$ & & & & $x$ & & $X^{*}$ \\
\hline $\begin{array}{r}\text { Weekly volume of } \\
\text { gastric residuals }\end{array}$ & & & & $x$ & & $X^{*}$ \\
\hline $\begin{array}{r}\text { Weekly n. of } \\
\text { apneas }\end{array}$ & & & & $x$ & & $X^{*}$ \\
\hline $\begin{array}{r}\text { Serious adverse } \\
\text { events }\end{array}$ & & & $x$ & $x$ & X & $X$ \\
\hline
\end{tabular}

Fig. 4 Trial flow-chart according to SPIRIT guidelines [57]. Black squares indicate timepoints when the intervention will surely be provided, while grey squares indicate a time point (36 weeks' postconceptional age) when the intervention may be provided unless it has been interrupted earlier. All assessments will be performed at the NICU discharge apart from the diagnosis of BPD (that requires evaluation of at 36 weeks' postconceptional age [24]), safety outcomes and serious adverse events

typical for MAS [55]. Neonatal ARDS: defined as per the international Montreux definition [52].

Ventilator free days defined as the number of days spent in the NICU without IMV. One point is given for each day during the NICU stay that patients are both alive and free of mechanical ventilation; in case of death, zero days will be given [56].

\section{Guidelines for protocol preparation}

The protocol has been prepared according to Standard Protocol Items: Recommendations for Interventional Trials (SPIRIT) guidelines [57], adapting them as much as possible to the field of neonatal ventilation trials. Figure 4 illustrates SPIRIT guidelines for our protocol. 


\section{Abbreviations}

ARDS: Acute respiratory distress syndrome; BPD: Bronchopulmonary dysplasia; Cl: Confidence interval; CPAP: Continuous positive airway pressure; CRIB-II: Critical risk index for babies-II; GA: Gestational age; IMV: Invasive mechanical; IVH: Intraventricular hemorrhage; MAS: Meconium aspiration syndrome; NEC: Necrotizing enterocolitis; NHFOV: Noninvasive high frequency oscillatory ventilation; NICU: Neonatal intensive care unit; NIPPV: Noninvasive positive pressure ventilation; OR: Odds ratio; Paw: Mean airway pressure; PDA: Patent ductus arteriosus; PIPP: Premature Infant Pain Profile; RDS: Respiratory distress syndrome; ROP: Retinopathy of prematurity; SIRS: Systemic inflammatory response syndrome; SPIRIT: Standard Protocol Items: Recommendations for Interventional Trials

\section{Acknowledgements \\ NASal OscillatioN post-Extubation (NASONE) study group}

A total of 69 Chinese NICUs and 114 investigators members of the study group.

1.Principal Investigator: Shi Yuan*, Jianhui Wang, Department of Neonatology, Children's Hospital of Chongqing Medical University, Ministry of Education Key Laboratory of Child Development and Disorders, Key Laboratory of Pediatrics in Chongqing; Department of Pediatrics, Daping hospital of Third Military Medical University. Chongqing, 400042, China. 2.Chen Long, Department of Pediatrics, Daping hospital of Third Military Medical University, Chongqing, 400042, China.

3.Xingwang Zhu, Huanhuan Li, Department of Neonatology JiulongpoPeople's Hospital; Department of Neonatology, Children's Hospital of Chongqing Medical University,Ministry of Education Key Laboratory of Child Development and Disorders, Key Laboratory of Pediatrics in Chongqing. Chongqing, 400042,China.

4. Xiaoyun Zhong, Sijie Song, Department of neonatology, Chongqing Women and Children Health Hospital. Chongqing, 401147, China.

5.Zhang Lan, Department of Pediatrics, Anhui province affiliated Hospital of Anhui medical university. Heifei, Anhui, 230000, China.

6.Li Li, Department of Neonatology, Children's Hospital of The Capital Institute of Pediatrics. Beijing, 100020, China.

7. Huiqiang Liu, Xiaomei Tong, Department of Pediatrics, Third Hospital, Peking University. Beijing, 100191, China.

8.Xiaojing Xu, LiFeng Cui, Department of Neonatology, the first affiliated hospital of Tsinghua University. Beijing, 1000016, China.

9.Ming Yi, Zhoujie Peng, Department of Neonatology, Women and children's hospital, branch of Chongqing sanxia central hospital. Wanzhou, Chongqing, 404000, China.

10. Li Jie, Department of Obstetrics and Gynecology, The First Affiliated Hospital of Chongqing Medical University. Chongqing, 400000,China. 11.Dongmei Chen, Weifeng Zhang, Department of Neonatal Intensive Care Unit, Quanzhou Children's Hospital. Quanzhou,Fujian,362000,China 12.Xinzhu Lin, Department of Neonatology, Maternal and Child Health Care Hospital of Xiamen. Xiamen,Fujian,361001, China.

13.Wang Bin, Neonatology and Pediatrics Department, Zhujiang Hospital, Southern Medical University. Guangzhou,Guangdong, 510000, China. 14.Weimin Huang, Guangliang Bi, Department of Neonatology, Nanfang Hospital, Southern Medical University. Guangzhou, Guangdong,510515, China 15.Shaoru He, Yumei Liu, Department of Neonatology, Guangdong Academy of Medical Science/Guangdong General Hospital. Guangzhou,Guangdong,510080, China,

16.Yang Jie, Gao Weiwei, Department of Neonatology, Guangdong Women and Children Hospital. Guangzhou, Guangdong,510010,China.

17.Wuhua Liang, Yaoxun Wu, Department of Neonatology, Women and Children's Health Hospital of Yulin. Yulin, Guangxi Zhuang Autonomous Region, 537000,China. 18.Xinnian Pan, Qiufen Wei, Department of Neonatology, Maternal and Children health care hospital of Guangxi Zhuang Autonomous Region. Nanning, Guangxi Zhuang Autonomous Region 530003,China .

19.Yujun Chen, Bingmei Wei, Department of Pediatrics, the Second Affiliated Hospital of Guangxi Medical University. Nanning, Guangxi Zhuang Autonomous Region,530007, China

20.Ling Liu, Department of Neonatology, Guiyang Maternal and Child Health Care Hospital. Guiyang, Guizhou, 550003,China.

21.Xinghui Zheng, Department of Neonatology, The first people's Hospital of Zunyi. Zunyi, Guizhou, 563000,China.

22.Ding Xu, Wang Fan, Department of Neonatology, the Second Hospital of Lanzhou University. Lanzhou, Gansu, 730030, China.
23.Bin Yi, Jingyun Shi, Department of neonatology, GanSu provencial Maternity and Child Care hospital. Lanzhou, GanSu, 730050,China. 24.Yuning Li, Li Jiang, Department of Pediatrics, The First Hospital of Lanzou University. Lanzhou,Gansu,730000,China.

25. Chunming Jiang, Department of Neonatology, The First Affiliated Hospital of Harbin Medical University. Harbin, Heilongjiang, 150000, China.

26.Chenghe Tang, Department of Neonatology, The First Affiliated Hospital of Xinxiang Medical University. Xingxiang, Henan, 453100, China.

27.Hong Xiong, Huiqing Sun, Wenqing Kang, Dapeng Liu, Neonatal Intensive

Care Unit and Department of Neonatology, Zhengzhou Children's Hospital, the Children's Hospital of Zhengzhou University. Zhengzhou,Henan, 450018, China. 28.Falin Xu, Department of Neonatology, The Third affiliated Hospital of Zhengzhou University. Zhengzhou, Henan 450052, China.

29.Xing Kaihui, Yang Ning, Department of Neonatal Intensive Care Unit, the Maternal and child health hospital of Hainan province. Haikou, Hainan, 570206, China. 30.Fang Liu, Shaoguang Lv, Department of Neonatal Intensive Care Unit, Bethune International Peace Hospital. Shijiazhuang, Hebei,050082, China. 31.Liu Hanchu, Yuan Wenchao, Department of Neonatology, Wuhan Children's Hospital, Tongji Medical College, Huazhong University of Science \& Technology. Wuhan, Hubei,43000, China.

32.Rui Cheng, Shen Xian, Department of Neonatology, Children's Hospital of Nanjing Medical University. Nanjing, Jiangsu, 210008, China.

33.Hui Wu, Department of Neonatology, the First Hospital of Jilin University. Changchun, Jilin,130021, China.

34.Laishuan Wang, Department of neonatology, Children's hospital of Fudan University. Shanghai, 201102, China.

35.Zhenying Yang, Zhang Xiao, Department of Neonatology, Maternal and Children's Healthcare Hospital of Taian. Taian,Shandong,271000,China. 36. Xue Jiang, Department of neonatal intensive care unit, the second hospital of Shandong University. Jinan, Shandong, 250033, China.

37.Zhankui Li, Department of Neonatology, North-West Women and Children's Hospital(maternal and Children's Healthcare Hospital of Shaanxi Province). Xian, Shanxi,710061, China.

38.Rong Ju, Wang Jin, Department of Neonatal Intensive Care Unit, the Women and Children's \Center Hospital of Chengdu. Chengdu, Sichuan, 610000, China. 39.Wenbin Dong, Department of Newborn Medicine, the Affiliated Hospital of Southwest Medical University. Luzhou,Sichuan,646000,China.

40. Ye Xiaoxiu, Department of Newborn Medicine,Hospital Affiliated to Southwest Medical University. Luzhou, Sichuan,646000, China.

41.Benqing Wu. Department of Neonatology, Shenzhen People's hospital/the Second Medical College of Jinan

University.Shenzhen, Guangdong,518020,China.

42.Zheng Jun, Tian Xiuying, Department of Neonatology, Tianjin Central Hospital of Obstetrics and Gynecology. Tianjin,300100, China. 43.Mingxia Li, Yanping Zhu, Department of Neonatology, the first affiliated hospital of Xinjiang Medical University. Urumqi, Xinjiang Uygur Autonomous Region ,830011, China.

44.Nuerya Rejiafu, Li Long, Department of Neonatology, People's Hospital of Xinjiang Uygur Autonomous Region. Urumqi, Xinjiang Uygur Autonomous Region 830001, China.

45.Yangfang Li, Canlin He, Department of neonatology, children's hospital of Kunming medical university. Kunming,Yunnan,650228,China.

46.Li Li, HongYing Mi, Pediatric Department, the first people's hospital of Yunnan province. Kunming,Yunnan, 650032, China.

47.Liang Kun, Department of Pediatrics, the first affiliated hospital of Kunming Medical University. Kunming,Yunnan,650032,China.

48.Hong Cao, Linglin Xia, Department of Neonatal Intensive Care Unit, Yan'an Hospital Affiliated to Kunming Medical University.

Kunming,Yunnan,650032, China.

49.Chuanfeng Li, Department of Neonatology, Women and Children's Health Hospital of Qujing. Qujing,Yunnan,655000,China.

50.Zhaoging Yin, Su Le, Department of neonatology, The people's hospital of Dehong autonomous prefecture. Mangshi, Yunnan, 678400, China.

51.Yanxiang Cheng, Department of Neonatology, Yinchuan No.1 people's Hospital Affiliated of Ningxia Medical University. Yinchuan, Ningxia, 750000 , China.

52.Liping Shi, Wang Chenhong, Department of Neonatal Intensive Care Unit, The Children's Hospital of Zhejiang University School of Medicine. Hangzhou, Zhejiang,310000, China.

53.Jiajun Zhu, Department of Neonatology, Women's Hospital, School of Medicine, Zhejiang University. Hangzhou,Zhejiang,310000,China. 
54.Zhang Xuefeng, Department of Neonatology, 302 Military hospital of china, People Liberation Army of China. Beijing,100000, China.

55.XiRong Gao, Bo Lv, Department of Neonatology, Hunan Children's hospital. Changsha, Hunan,410007, China.

56.Liu Chongde, Wang Xiaorong, Department of Neonatology,Women and Children's Hospital of Qinghai. Xining, Qinghai,810007,China.

57.Chen Liping, Li Lin, Department of Neonatology, Jiangxi Provincial Children's Hospital. Nanchang,Jiangxi,330006,China.

58.Zhang Chunli, Department of Neonatology,Inner Mongolia People's Hospital. Hohhot, Inner Mongolia,010000,China.

59.Chen Jia, Department of Neonatology, Mianyang central hospital. Mianyang, Sichuan,621000, China.

60.Qiyu Li, Department of Pediatrics, 202 Military hospital of People's Liberation Army of China, Shenyang, Liaoning, 110000,China.

61.Lv Qin, Yanhong Li, Infant Intensive Care Unit, Ningbo women and children hospital. Ningbo, Zhejiang,315010, China.

62.Yong Ji,bYanjiang Chen, NICU, Children's hospital of Shanxi. Taiyuan, Shanxi,030000, China.

63.Jianhua Sun, Jun Bu, Department of Neonatology, Shanghai Children's Medical Center. Shanghai,200127, China.

64.Danni Zhong, Zongyan Gao, Department of Pediatrics,The First Affiliated Hospital of Guangxi Medical University. Nanning, Guangxi Zhuang Autonomous Region,530021, China.

65.Han Shuping, Xiaohui Chen, Department of Pediatrics, Obstetrics and Gynecology Hospital of Affiliated to Nanjing Medical University. Nanjing, Jiangsu, 210001, China.

66. Caiyun Gao, Hongbin Zhu, Pediatric Department, Qinhuangdao maternal and child health care hospital. Qinhuangdao, Hebei, 066000, China. 67.Zhenguang Li, Hongwei Wu, Pediatric Department, Xuzhou Children Hospital. Xuzhou, Jingsu, 221000, China.

68.Xiuyong Cheng, Pediatric Department, The first affiliated hospital of Zhengzhou university, Zhengzhou, Henan, 450000, China.

69.Juhua Li, Department of Pediatrics, Xianyang children's Hospital. Xianyang, Shanxi, 712021, China.

\section{Authors' contributions}

YS and DDL equally contributed to the protocol preparation and the paper writing; they read and approved the final manuscript.

\section{Funding}

The trial is initiated and planned by the researchers and funded by Scientific Research Projects unit of Chongqing (Project- cstc2016shms-ztzx13001). Only this public funding will be used for the study, teleconferences, international panel meeting and for publication costs.

This is a non-promoted study and investigators did not receive and will not receive any fee from any industry. In no way industry or commercially interested subjects will have access to the data, before their publication. The study has not received any grant or support from any commercial company/organization. The study only received public funding from Chongqing Government.

\section{Availability of data and materials}

Not applicable for the study protocol. The datasets used and/or analyzed during the trial will be made available from the corresponding author on reasonable request.

\section{Ethics approval and consent to participate}

The trial has been approved by the Ethical Committee of the Third Affiliated Hospital of Third Military Medical University - Human Research Protection Program (with the n.201721) and informed consent will be obtained from parents or guardians antenatally or upon NICU admission. The trial is registered in the clinicaltrial.gov registry (ID:NCT03181958) and will be performed in accordance with the approved guidelines and regulations of the participating institutions.

Any modification to the protocol which may impact on the conduct of the study, potential benefit of the patient or may affect patient safety, including changes of study objectives, study design, patient population, sample sizes, study procedures, or significant administrative aspects will require a formal amendment to the protocol. Thus, they will have to be approved by all investigators and will require a new ethical approval.

\section{Consent for publication}

Not applicable

\section{Competing interests}

YS has no interest to declare DDL has received research support, travel grants and/or consultancy fee from Carefusion (now Vyaire), Acutronic and Getinge. Those are industries producing neonatal ventilators. These companies are not involved at all in the study and will not have any role in the conduct of the study; collection, management, analysis, and interpretation of the data; preparation, review, approval of the manuscript or decision to submit it for publication. Many different ventilators will be variably used in the trial (see Methods and design).

\section{Author details}

'Department of Neonatology, Children's Hospital of Chongqing Medical University, Ministry of Education Key Laboratory of Child Development and Disorders, China International Science and Technology Cooperation Base of Child Development and Critical Disorders, Chongqing 400014, China.

2Division of Pediatrics and Neonatal Critical Care, "A.Béclère" Medical Center, South Paris University Hospitals, AP-HP, Paris, France. ${ }^{3}$ Physiopathology and Therapeutic Innovation Unit-INSERM U999, South Paris-Saclay University, Paris, France. ${ }^{4}$ Institute of Anesthesiology and Critical Care, Catholic University of the Sacred Heart, Rome, Italy.

\section{Received: 23 October 2017 Accepted: 12 July 2019}

Published online: 26 July 2019

\section{References}

1. Lemons JA, Bauer CR, Oh W, Korones SB, Papile LA, Stoll BJ, Verter J, Temprosa M, Wright LL, Ehrenkranz RA, Fanaroff AA, Stark A, Carlo W, Tyson JE, Donovan EF, Shankaran S, Stevenson DK. Very low birth weight infant outcomes of the NICHD neonatal research network. Pediatrics. 2001;107:1-8.

2. Schmölzer GM, Kumar M, Pichler G, Aziz K, O'Reilly M, Cheung PY. Noninvasive versus invasive respiratory support in preterm infants at birth: systematic review and meta-analysis. BMJ. 2013;347:f5980.

3. Subramaniam P, Ho JJ, Davis PG. Prophylactic nasal continuous positive airway pressure for preventing morbidity and mortality in very preterm infants. Cochrane Database Syst Rev. 2016;6:CD001243.

4. De Paoli AG, Davis PG, Faber B, Morley CJ. Devices and pressure sources for administration of nasal continuous positive airway pressure (NCPAP) in preterm neonates. Cochrane Database Syst Rev. 2008;1:CD002977.

5. Kieran EA, Twomey AR, Molloy EJ, Murphy JF, O'Donnell CP. Randomized trial of prongs or mask for nasal continuous positive airway pressure in preterm infants. Pediatrics. 2012;130:e1170-6.

6. Sweet DG, Carnielli V, Greisen G, Hallman M, Ozek E, Plavka R, Saugstad OD, Simeoni U, Speer CP, Vento M, Visser GH, Halliday HL. European Consensus Guidelines on the Management of Respiratory Distress Syndrome - 2016 Update. Neonatology. 2017;111:107-25.

7. Stefanescu BM, Murphy WP, Hansell BJ, Fuloria M, Morgan TM, Aschner JL. A randomized, controlled trial comparing two different continuous positive airway pressure systems for the successful extubation of extremely low birth weight infants. Pediatrics. 2003;112:1031-8.

8. Barrington KJ, Bull D, Finer NN. Randomized trial of nasal synchronized intermittent mandatory ventilation compared with continuous positive airway pressure after extubation of very low birth weight infants. Pediatrics. 2001;107:638-41.

9. Davis PG, Henderson-Smart DJ. Nasal continuous positive airways pressure immediately after extubation for preventing morbidity in preterm infants. Cochrane Database Syst Rev. 2003;2:CD000143.

10. Greenough A. Long-term pulmonary outcome in the preterm infant. Neonatology. 2008;93:324-7.

11. Chien YH, Tsao PN, Chou HC, Tang JR, Tsou Kl. Rehospitalization of extremely-low-birth-weight infants in first 2 years of life. Early Hum Dev. 2002;66:33-40.

12. Lemyre B, Davis PG, De Paoli AG, Kirpalani H. Nasal intermittent positive pressure ventilation (NIPPV) versus nasal continuous positive airway pressure (NCPAP) for preterm neonates after extubation. Cochrane Database Syst Rev. 2017;2:CD003212.

13. De Luca D, Dell'Orto V. Non-invasive high-frequency oscillatory ventilation in neonates: review of physiology, biology and clinical data. Arch Dis Child Fetal Neonatal Ed. 2016. https://doi.org/10.1136/archdischild-2016-310664. 
14. Czernik C, Schmalisch G, Bührer C, Proquitté $H$. Weaning of neonates from mechanical ventilation by use of nasopharyngeal high frequency oscillatory ventilation: a preliminary study. J Matern Fetal Neonatal Med. 2012;25:374-8.

15. Mukerii A, Sarmiento K, Lee B, Hassall K, Shah V. Non-invasive highfrequency ventilation versus bi-phasic continuous positive airway pressure (BP-CPAP) following CPAP failure in infants <1250 g: a pilot randomized controlled trial. J Perinatol. 2017;37:49-53.

16. De Luca D. Noninvasive high-frequency ventilation and the errors from the past: designing simple trials neglecting complex respiratory physiology. J Perinatol. 2017;37:1065-6.

17. Fisher HS, Bohlin K, Bührer C, Schmalisch G, Cremer M, Reiss I, Czernik C. Nasal high-frequency oscillation ventilation in neonates: a survey in five European countries. Eur J Pediatr. 2015;174:465-71.

18. Moher D, Hopewell S, Schulz KF, Montori V, Gøtzsche PC, Evereaux PJ, Elbourne D, Egger M, Altman DG. CONSORT 2010 explanation and elaboration: updated guidelines for reporting parallel group randomised trials. BMJ. 2010 Mar 23:340:c869. https://doi.org/10.1136/bmj.c869.

19. Restrepo RD, Hirst KR, Wittnebel L, Wettstein R. AARC clinical practice guideline: transcutaneous monitoring of carbon dioxide and oxygen: 2012 Respir Care. 2012;57:1955-62.

20. Silverman WC, Anderson DH. Controlled clinical trial on effects of water mist on obstructive respiratory signs, death rate and necropsy findings among premature infants. Pediatrics. 1956;17:1-4.

21. Papile LA, Munsick-Bruno G, Schaefer A. Relationship of cerebral intraventricular hemorrhage and early childhood neurologic handicaps. J Pediatr. 1983:103:273-7.

22. Watkins AM, West CR, Cooke RW. Blood pressure and cerebral hemorrhage and ischaemia in very low birth weight infants. Early Hum Dev. 1989;19:103-10.

23. De Luca D, Romain O, Yousef N, Andriamanamirijaa D, Shankar-Aguilera S, Walls $E$, Sgaggero B, Aube N, Tissières P. Monitorages physiopathologiques en reanimation néonatale. J Ped Puericul. 2015;28:276-300 [Article in French].

24. Jobe AH, Bancalari E. Bronchopulmonary dysplasia. Am J Respir Crit Care Med. 2001;163:1723-9.

25. International Committee for the Classification of Retinopathy of Prematurity. The international classification of retinopathy of prematurity revisited. Arch Ophthalmol. 2005;123:991-9.

26. Stoll BJ. Epidemiology of necrotizing enterocolitis. Clin Perinatol. 1994;21:205-18.

27. Fischer C, Bertelle V, Hohlfeld J, Forcada-Guex M, Stadelmann-Diaw C, Tolsa JF. Nasal trauma due to continuous positive airway pressure in neonates. Arch Dis Child Fetal Neonatal Ed. 2010:95:F447-51.

28. Stevens $B$, Johnston C, Petryshen P, Taddio A. Premature infant pain profile :developmend and initial validation. Clin J Pain. 1996:12:13-2.

29. De Paoli AG, Davis PG, Faber B, Morley CJ. Devices and pressure sources for administration of nasal continuous positive airway pressure (NCPAP) in preterm neonates. Cochrane Database Syst Rev. 2002;3:CD002977.

30. Roberts CT, Davis PG, Owen LS. Neonatal non-invasive respiratory support: synchronised NIPPV, non-synchronised NIPPV or bi-level CPAP: what is the evidence in 2013? Neonatology. 2013;104:203-9.

31. Mukerii A, Belik J. Neonatal nasal intermittent positive pressure ventilation efficacy and lung pressure transmission. J Perinatol. 2015;35:716-9.

32. Gerdes JS, Sivieri EM, Abbasi S. Factors influencing delivered mean aimay pressure during nasal CPAP with the RAM cannula. Pediatr Pulmonol. 2016;51:60-9.

33. De Jaegere $A$, van Veenendaal MB, Michiels $A$, van Kaam AH. Lung recruitment using oxygenation during openlunghigh-frequency ventilation in preterm infants. Am J Respir Crit Care Med. 2006;174:639-45.

34. De Luca D, Piastra M, Pietrini D, Conti G. Effect of amplitude and inspiratory time in a bench model of non-invasive HFOV through nasal prongs. Pediatr Pulmonol. 2012;47:1012-8.

35. De Luca D, Carnielli VP, Conti G, Piastra M. Noninvasive high frequency oscillatory ventilation through nasal prongs: bench evaluation of efficacy and mechanics. Intensive Care Med. 2010;36:2094-100.

36. Mukerji A, Finelli M, Belik J. Nasal high-frequency oscillation for lung carbon dioxide clearance in the newborn. Neonatology. 2013;103:161-5.

37. De Luca D, Costa R, Visconti F, Piastra M, Conti G. Oscillation transmission and volume delivery during face mask-delivered HFOV in infants: Bench and in vivo study. Pediatr Pulmonol. 2016;51:705-12.

38. Centorrino R, Dell'Orto V, Gitto E, Conti G, De Luca D. Mechanics of nasal mask-delivered HFOV in neonates: a physiologic study. Pediatr Pulmonol. 2019; accepted - in press.

39. Dell'Orto V, Centorrino R, Shankar-Aguilera S, Ben-Ammar R, Yousef N, De Luca D. Noninvasive high frequency oscillatory ventilation versus noninvasive intermittent positive pressure ventilation for preterm babies developing BPD: a pilot study. E-PAS 2018 in press (Abstract to the Pediatric Academic Societies meeting 2018).

40. Ramanathan R, Sekar KC, Rasmussen M, Bhatia J, Soll RF. Nasal intermittent positive pressure ventilation after surfactant treatment for respiratory distress syndrome in preterm infants $<=30$ weeks' gestation: a randomized, controlled trial. J Perinatol. 2012;32:336-43.

41. Faul F, Erdfelder E, Lang AG, Buchner A. G*power 3: a flexible statistical power analysis program for the social, behavioral, and biomedical sciences. Behav Res Methods. 2003;39:175-91.

42. Parry G, Tucker J, Tarnow-Mordi W. UK neonatal staffing study collaborative group. CRIB II: an update of the clinical risk index for babies score. Lancet. 2003:24(361):1789-91.

43. Allison PD. Logistic regression using the SAS system. Cary: SAS Institute Ed; 1999.

44. De Luca D, Harrison DA, Peters MJ. 'Lumping or splitting' in paediatric acute respiratory distress syndrome (PARDS). Intensive Care Med. 2018;44:1548-50

45. Thorpe KE, Zwarenstein M, Oxman AD, Treweek S, Furberg CD, Altman DG, Tunis S, Bergel E, Harvey I, Magid DJ, Chalkidou K. A pragmatic explanatory continuum indicator summary (PRECIS): a tool to help trial designers. J Clin Epidemiol. 2009:62:464-75.

46. Sweet DG, Carnielli V, Greisen G, Hallman M, Ozek E, Te Pas A, Plavka R, Roehr CC, Saugstad OD, Simeoni U, Speer CP, Vento M, Visser GHA, Halliday $\mathrm{HL}$. European consensus guidelines on the Management of Respiratory Distress Syndrome - 2019 update. Neonatology. 2019;115:432-51.

47. Frerichs I, Amato MB, van Kaam AH, Tingay DG, Zhao Z, Grychtol B, Bodenstein M, Gagnon H, Böhm SH, Teschner E, Stenqvist O, Mauri T, Torsani V, Camporota L, Schibler A, Wolf GK, Gommers D, Leonhardt S. Adler a; TREND study group. Chest electrical impedance tomography examination, data analysis, terminology, clinical use and recommendations: consensus statement of the TRanslational ElT developmeNt stuDy group. Thorax. 2017;72:83-93.

48. Wolf GK, Arnold JH. Noninvasive assessment of lung volume: respiratory inductance plethysmography and electrical impedance tomography. Crit Care Med. 2005;33(3 Suppl):S163-9.

49. Raimondi F, Yousef N, Migliaro F, Capasso L, De Luca D. Point-of-care lung ultrasound in neonatology: classification into descriptive and functional applications. Pediatr Res. 2018 Jul 20. https://doi.org/10.1038/s41390-0180114-9 [Epub ahead of print].

50. Li J, Li X, Huang X, Zhang Z. Noninvasive high-frequency oscillatory ventilation as respiratory support in preterm infants: a meta-analysis of randomized controlled trials. Respir Res. 2019;20(1):58. https://doi.org/10.1186/s12931-019-1023-0.

51. Shankar-Aguilera S, Taveira M, De Luca D. Neonatal ventilation trials need specific funding. Lancet Respir Med. 2014;2:867-9.

52. De Luca D, van Kaam AH, Tingay DG, Courtney SE, Danhaive O, Carnielli VP, Zimmermann U, Kneyber MC, Tissieres P, Brierley J, Conti G, Pillow JJ, Rimensberger PC. The Montreux definition of neonatal ARDS: biological and clinical background behind the description of a new entity. Lancet Respir Med. 2017;5:657-66.

53. De Luca D, Baroni S, Vento G, Piastra M, Pietrini D, Romitelli F, Capoluongo E, Romagnoli C, Conti G, Zecca E. Secretory phospholipase A2 and neonatal respiratory distress: pilot study on broncho-alveolar lavage. Intensive Care Med. 2008;34:1858-64.

54. Goldstein B, Giroir B, Randolph A. International Consensus Conference on Pediatric Sepsis. International pediatric sepsis consensus conference: definitions for sepsis and organ dysfunction in pediatrics. Pediatr Crit Care Med. 2005;6:2-8.

55. Piastra M, Yousef N, Brat R, Manzoni P, Mokhtari M, De Luca D. Lung ultrasound findings in meconium aspiration syndrome. Early Hum Dev. 2014;90(Suppl2):S41-3.

56. Contentin L, Ehrmann S, Giraudeau B. Heterogeneity in the definition of mechanical ventilation duration and ventilator-free days. Am J Respir Crit Care Med. 2014;15(189):998-1002.

57. Chan A-W, Tetzlaff JM, Altman DG, Laupacis A, Gøtzsche PC, Krleža-Jerić K, Hróbjartsson A, Mann H, Dickersin K, Berlin J, Doré C, Parulekar W, Summerskill W, Groves T, Schulz K, Sox H, Rockhold FW, Rennie D, Moher D. SPIRIT 2013 statement: defining standard protocol items for clinical trials. Ann Intern Med. 2013;158:200-7.

\section{Publisher's Note}

Springer Nature remains neutral with regard to jurisdictional claims in published maps and institutional affiliations. 